\title{
Optical doping and damage formation in AIN by Eu implantation
}

\author{
K. Lorenz, ${ }^{1, a)}$ E. Alves,${ }^{1}$ F. Gloux,${ }^{2}$ P. Ruterana, ${ }^{2}$ M. Peres,${ }^{3}$ A. J. Neves, ${ }^{3}$ and \\ T. Monteiro ${ }^{3}$ \\ ${ }^{1}$ Instituto Tecnológico e Nuclear, Estrada Nacional 10, 2686-953 Sacavém, Portugal and CFNUL, Av. Prof. \\ Gama Pinto, 1649-003 Lisboa, Portugal \\ ${ }^{2}$ CIMAP, UMR 6252, CNRS-ENSICAEN-CEA-UCBN, 6 Boulevard du Marechal Juin, 14050 Caen Cedex, \\ France \\ ${ }^{3}$ Departamento de Física and I3N, Universidade de Aveiro, 3810-193 Aveiro, Portugal
}

(Received 23 September 2009; accepted 14 December 2009; published online 28 January 2010)

\begin{abstract}
AlN films grown on sapphire were implanted with $300 \mathrm{keV} \mathrm{Eu}$ ions to fluences from $3 \times 10^{14}$ to $1.4 \times 10^{17}$ atoms $/ \mathrm{cm}^{2}$ in two different geometries: "channeled" along the $c$-axis and "random" with a $10^{\circ}$ angle between the ion beam and the surface normal. A detailed study of implantation damage accumulation is presented. Strong ion channeling effects are observed leading to significantly decreased damage levels for the channeled implantation within the entire fluence range. For random implantation, a buried amorphous layer is formed at the highest fluences. Red Eu-related photoluminescence at room temperature is observed in all samples with highest intensities for low damage samples (low fluence and channeled implantation) after annealing. Implantation damage, once formed, is shown to be stable up to very high temperatures. (C) 2010 American Institute of Physics. [doi:10.1063/1.3291100]
\end{abstract}

\section{INTRODUCTION}

Emissions from optically active rare earth (RE) ions span a wide range of the electromagnetic spectrum from UV to IR including the entire visible spectrum. Since Favennec et $a l^{1}{ }^{1}$ pointed out that optical quenching of luminescence is found to decrease with increasing band gap, $\mathrm{GaN}$ and to a lesser extent $\mathrm{AlGaN}$ and $\mathrm{AlN}$ have been intensively studied as host material for RE ions. ${ }^{2} \mathrm{GaN}$ :RE based electroluminescent devices in all three primary colors and even laser action in Eu-doped GaN were reported. ${ }^{3,4} \mathrm{Eu}$ and Tm luminescence intensity was found to increase in $\mathrm{Al}_{\mathrm{x}} \mathrm{Ga}_{1-\mathrm{x}} \mathrm{N}$ when the $\mathrm{Al}$ content was raised from $\mathrm{x}=0$ to $\mathrm{x}=0.5 .^{5}$ Due to the large band gap of AIN ( $6.2 \mathrm{eV})$ a low thermal quenching of the $4 \mathrm{f}^{\mathrm{n}}$ intraionic transitions is expected and energetically high lying RE levels can be exploited. RE emissions in the visible and UV spectral region were reported for $\mathrm{Pr}, \mathrm{Eu}, \mathrm{Tb}, \mathrm{Tm}$, and Gd doped AlN. ${ }^{6-11}$ Equally interesting are the recently reported magnetic properties of RE implanted AlN showing ferromagnetism at room temperature (RT). ${ }^{12}$ Both optical and magnetic properties of implanted AlN will be strongly dependent on the structural quality of the films and defects produced during the implantation. Few detailed studies on implantation damage buildup and postimplant annealing are published. ${ }^{13-16}$ In all these studies random implantation was applied, meaning that the implantation was performed along an off-axis direction in order to suppress channeling effects. The authors pointed to the strong irradiation hardness due to efficient dynamic annealing effects. However, once formed, implantation damage is highly stable and negligible lattice recovery was reported for rapid thermal annealing at $1000{ }^{\circ} \mathrm{C}$ (Ref. 14) or $60 \mathrm{~min}$ annealing at $950{ }^{\circ} \mathrm{C} .{ }^{17}$ Therefore, it is of utmost importance to keep damage low already

\footnotetext{
a) Author to whom correspondence should be addressed. Electronic mail: lorenz@itn.pt.
}

during the implantation. In the case of GaN it was shown that implanting in a channeling geometry, where the ion beam is aligned with the GaN $c$-axis, can reduce the level of incorporated lattice damage. ${ }^{18,19}$

In this work, we present a comprehensive study on damage buildup in AlN upon Eu implantation in channeled and random geometry and report on the effect of implantation damage on the emission properties of optically active Eu ions. It is shown that channeled implantation can significantly reduce the damage formation rate and positively influence the activation of $\mathrm{RE}$ ions during high temperature annealing.

\section{EXPERIMENTAL DETAILS}

Commercial (Technologies and Devices International Inc.) $\sim 3 \mu \mathrm{m}$ thick AlN films grown by hydride vapor phase epitaxy on (0001) sapphire substrates were implanted at $\mathrm{RT}$ with $\mathrm{Eu}$ ions to fluences from $3 \times 10^{14}$ to 1.4 $\times 10^{17}$ atoms $/ \mathrm{cm}^{2}$ at an energy of $300 \mathrm{keV}$. The implantation was either performed in channeled geometry with the ion beam aligned with the $c$-axis or in random geometry where the surface normal was inclined away from the beam direction by $10^{\circ}$. Postimplant annealing was performed in a tube furnace at $1300{ }^{\circ} \mathrm{C}$ for $20 \mathrm{~min}$ in nitrogen atmosphere; during the process an unimplanted piece of AlN had been placed face to face with the sample in order to protect the surface.

Rutherford backscattering/channeling (RBS/C) studies were performed with a $1 \mathrm{~mm}$ diameter collimated beam of 2 $\mathrm{MeV} \mathrm{He}{ }^{+}$ions. The backscattered particles were detected at $140^{\circ}$ and close to $180^{\circ}$ with respect to the incoming beam direction using silicon surface barrier detectors. "Aligned" spectra were taken along the GaN $c$-axis and "random" spectra were acquired by tilting the sample by $5^{\circ}$ and rotating it during the measurement. 


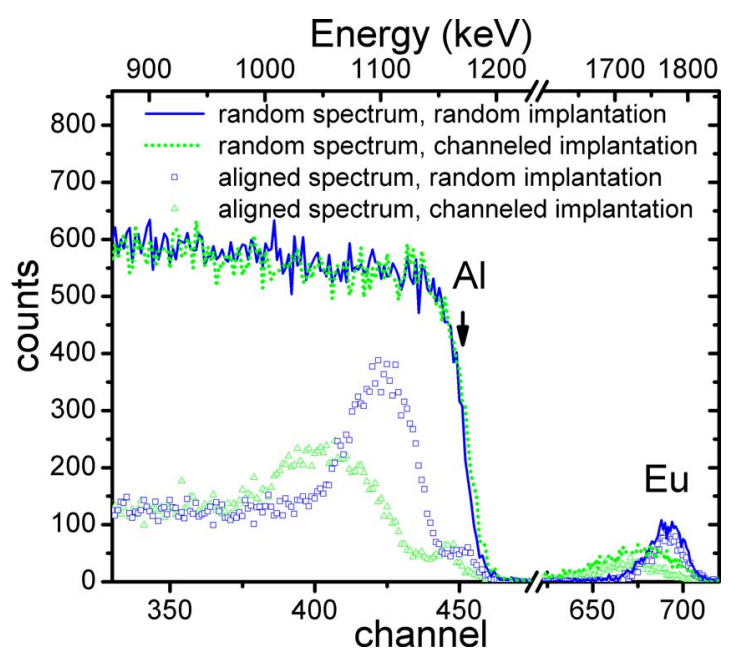

FIG. 1. (Color online) Random and $\langle 0001\rangle$ aligned RBS/C spectra after implantation of $300 \mathrm{keV} \mathrm{Eu}$ ions to a fluence of $3 \times 10^{15} \mathrm{atoms} / \mathrm{cm}^{2}$ in random and channeled geometry.

Transmission electron microscopy (TEM) observations of selected samples were performed with a JEOL 2010 instrument operating at $200 \mathrm{kV}$. The samples were thinned first by mechanical grinding to $100 \mu \mathrm{m}$ and then dimpled down to $10-20 \mu \mathrm{m}$ in the center. Electron transparency was achieved by ion milling with a final beam incidence of around $3^{\circ}$ and energy of $2.5 \mathrm{keV}$.

Optical characterization was performed by microphotoluminescence (micro-PL) at RT with excitation below the band gap of AlN using a $\mathrm{HeCd}$ laser. It was shown previously by PL excitation measurements that Eu emission from AlN can be excited below the band gap via two broad absorption bands around 270 and $350 \mathrm{~nm} \cdot{ }^{20}$

\section{RESULTS AND DISCUSSION}

Figure 1 shows the random and aligned RBS/C spectra for samples implanted to a fluence of $3 \times 10^{15}$ atoms $/ \mathrm{cm}^{2}$ in random and channeled geometry. RBS/C depth-profiling showed that the maximum Eu concentrations of 0.4 and 0.25 at. $\%$ are at depths of 50 and $90 \mathrm{~nm}$ for random and channeled implantation, respectively, with a channeling tail reaching deep into the sample for the latter. In the case of channeled implantation, the Eu and damage profile reach deeper into the sample and the maximum damage level is strongly reduced both due to the reduced direct collisions of the ions with the target atoms and the wider defect distribution. The defect profiles were extracted from the RBS/C spectra using the DICADA code to account for the contribution of dechanneling to the backscattering yield. ${ }^{21}$ Figure 2 presents selected defect profiles for random implantation. The relative defect levels in the Al sublattice at the maximum of the defect distributions as a function of the fluence are shown in Fig. 3. The fluence was also converted to displacements per atoms using the SRIM2008 (Ref. 22) Monte Carlo code with displacement energies of $50 \mathrm{keV}$ for both $\mathrm{Al}$ and $\mathrm{N}$ atoms. Note that only the damage in the Al-sublattice is measured in the present conditions. Jiang et $a l .{ }^{16}$ reported similar damage behaviors for the Al- and $\mathrm{N}$-sublattices.

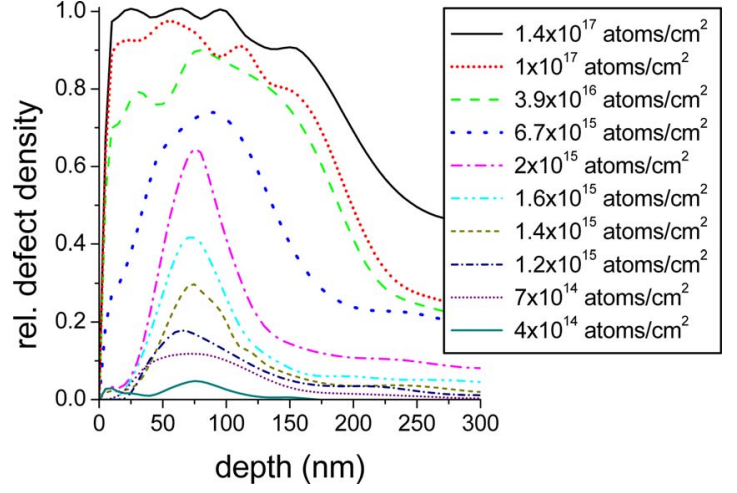

FIG. 2. (Color online) Selected defect profiles derived from the RBS/C spectra using the DICADA code for random implantation.

In a low fluence regime, the defect accumulation rate is low due to efficient dynamic annealing for both implantation geometries. This regime was also studied by Wendler and Wesch ${ }^{15}$ for implantation at $10 \mathrm{~K}$, where defect diffusion is low; the high resistance to radiation was explained by the strong bond strength of the material which was correlated with an atomic force constant determined from the longwave optical constants.

In an intermediate fluence regime, a strong increase in implantation damage is observed followed by a third regime where damage increases gradually but slowly. While the shape of the curves is similar for both implantation geometries, for channeled implantation, the onset of the different regimes is shifted toward significantly higher fluences and the damage level stays lower in the entire investigated fluence range. Only for random implantation does the relative damage reach unity for the highest fluences suggesting the formation of an amorphous layer.

The implantation damage was also studied by TEM. Figures 4(a) and 4(b) show images in 0002 and $10 \overline{1} 0$ weak beam conditions for a sample implanted to a fluence of 1.4 $\times 10^{15}$ atoms $/ \mathrm{cm}^{2}$ in random geometry. In 0002 weak beam conditions, the atomic displacements along the [0002] direction are revealed. The contrast may be due to point defect

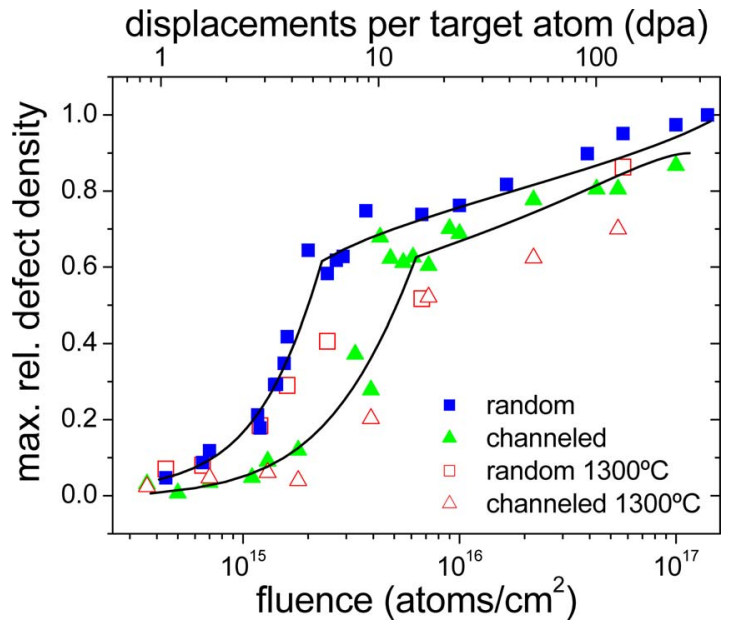

FIG. 3. (Color online) The relative disorder in the Al-sublattice at the peak of the damage distribution for random and channeled implantation before and after annealing at $1300^{\circ} \mathrm{C}$ as a function of the implantation fluence. 


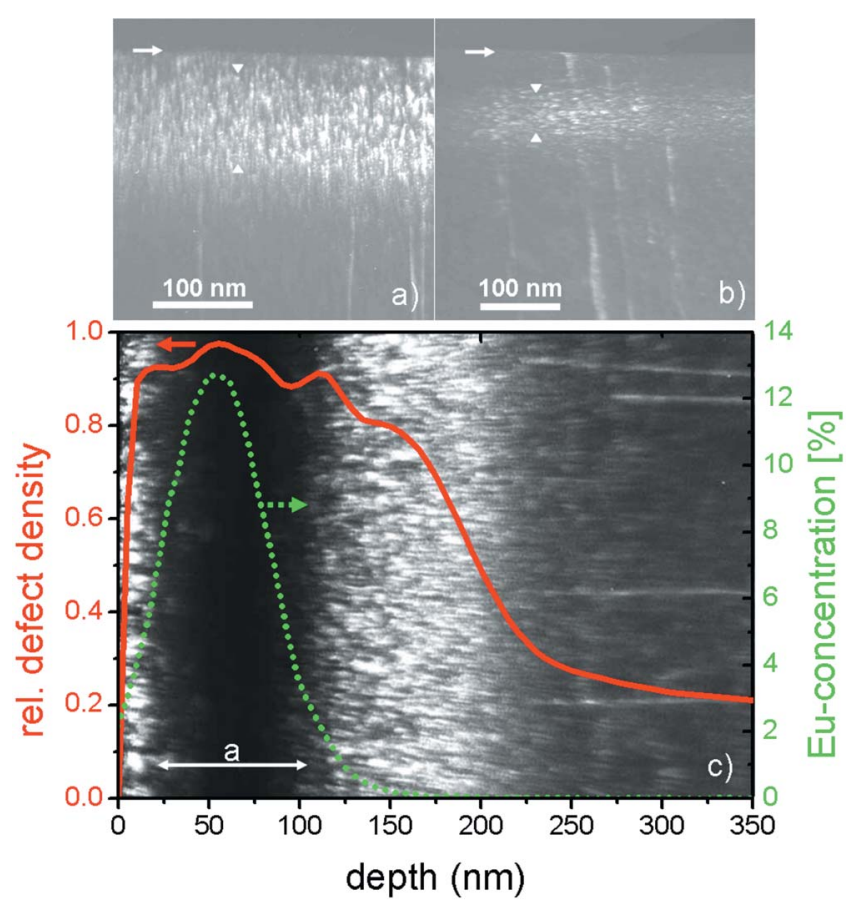

FIG. 4. (Color online) (a) TEM dark field weak beam image with $g=0002$ of AlN implanted with $1.4 \times 10^{15}$ atoms $/ \mathrm{cm}^{2} \mathrm{Eu}$ in random geometry. The surface is marked with a white horizontal arrow and the region with point defect clusters is indicated. (b) TEM dark field weak beam image of the same sample as in (a) with $g=10 \overline{1} 0$. The region with planar defects is indicated. (c) TEM dark field weak beam image with $g=0002$ of AlN implanted with $1 \times 10^{17}$ atoms $/ \mathrm{cm}^{2} \mathrm{Eu}$ in random geometry showing the formation of a buried amorphous layer marked with a. Overlaid to the TEM image are the Eu- and defect profiles determined by RBS/C.

clusters and/or other defects such as dislocation loops, or prismatic defects which have a component along the $c$-axis; the visible damaged region runs from near the surface to about $130 \mathrm{~nm}$ into the bulk. With $\mathrm{g}=10 \overline{1} 0$ the contrast is due to displacements that have taken place inside the basal planes, the corresponding defects being for example basal stacking faults. The region with such bright contrast is narrower and the surface region is seen to be nearly free of such defects. For higher fluences the contrast due to these defects is increasing with lower defect concentrations for channeled implantation. Similar defect signatures were observed for $\mathrm{Eu}$ implantation in the related semiconductor $\mathrm{GaN}^{23}$

At a fluence of $1 \times 10^{17}$ atoms $/ \mathrm{cm}^{2}$ in random geometry, the formation of a buried amorphous layer is observed as shown in the TEM image in Fig. 4(c). Electron diffraction patterns confirm the amorphous structure. Overlaid to the TEM image are the Eu- and defect profiles derived from the $\mathrm{RBS} / \mathrm{C}$ measurements. RBS/C spectra suggest that amorphisation starts close to the end of range of the Eu ions forming a buried amorphous layer which then extends toward the surface and the bulk of the crystal (see also Fig. 2). Furthermore, it is interesting to note that the RBS/C defect profile corresponds well to the TEM defect contrast. High defect levels are to be pointed out for deep regions in which the Eu-concentration is already low pointing to a diffusion of Al-interstitials. A diffusion of defects toward the AlN bulk during high fluence Au implantation was also observed by Jiang et al. ${ }^{16}$ and may be the reason why these authors re-

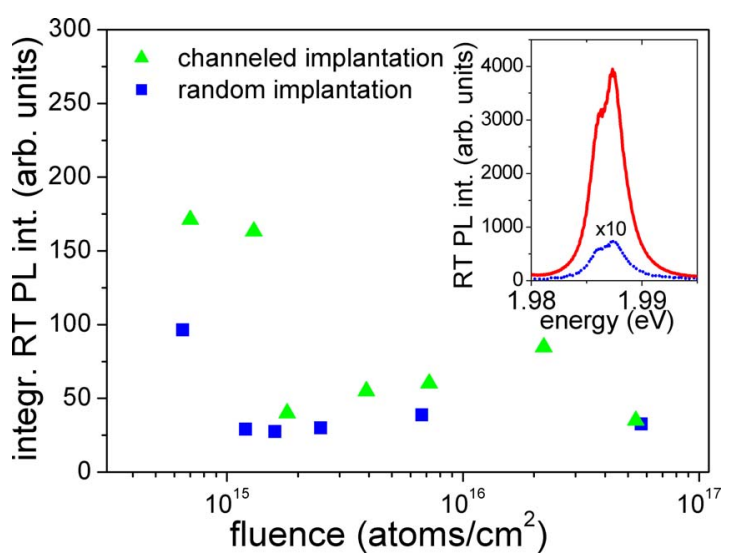

FIG. 5. (Color online) Integrated RT PL intensity of the Eu emission around $624 \mathrm{~nm}(1.987 \mathrm{eV})$ after annealing as a function of the fluence for channeled and random implantation. The inset shows typical RT PL spectra of an as-implanted sample (blue dotted line) and after annealing (red solid line).

ported a saturation of the defect level for high fluences. The peculiar gradual increase in implantation damage observed for the high fluence regime in Fig. 3 might be connected to chemical effects which may also play a role in the formation of the amorphous phase. Another study of $\mathrm{Au}$ implantation into AlN reports the breakdown of monocrystalline order but no amorphisation. ${ }^{14}$ The reason for this behavior may be the lower crystal quality of the as-grown material in this early study where grain boundaries or dislocations can reduce the diffusivity of point defects.

It is interesting to compare the present results to $\mathrm{Eu}$ implantation into the related semiconductor $\mathrm{GaN}$ under similar conditions. $^{24}$ The damage buildup curve for $\mathrm{GaN}$ is very similar with low damage accumulation for low fluences and a strong damage increase in the intermediate fluence regime. This strong increase in damage, however, starts at substantially lower fluences. For RT implantation, damage in the bulk of $\mathrm{GaN}$ saturates for high fluences, while an enhanced surface damage causes strong dechanneling and nullifies the positive effect of channeled implantation already for moderate fluences. ${ }^{19}$ In the case of AlN the maximum damage level is found close to the end of range of the implanted Eu, no preferred surface damage is observed and the ion channeling effect is preserved up to the highest studied fluences.

All samples show bright red Eu-related PL at RT already in the as-implanted state. After annealing at $1300{ }^{\circ} \mathrm{C}$ only very little structural recovery was observed (see damage levels in Fig. 3). Nevertheless, a strong increase in the Eurelated light emission is achieved (see inset in Fig. 5). Figure 5 presents the integrated PL intensity of the $\mathrm{Eu}$ emission lines around $\sim 624 \mathrm{~nm}$ arising from the ${ }^{5} \mathrm{D}_{0} \rightarrow{ }^{7} \mathrm{~F}_{2}$ intraionic $4 \mathrm{f}^{6}$ transitions after annealing. It is clearly observed that the PL intensity is higher for channeled implantation within the entire fluence range. Furthermore, despite low Eu concentrations we find highest PL intensities for the samples within the low fluence regime. PL decreases strongly when the fluence reaches a critical value of $\sim 1 \times 10^{15}$ atoms $/ \mathrm{cm}^{2}$ for random and $\sim 2 \times 10^{15}$ atoms $/ \mathrm{cm}^{2}$ for channeled implantation showing that implantation damage in AIN introduces nonradiative recombination paths. In good agreement with the structural results this drop of PL intensity occurs at 
higher fluences for channeled implantation and in the region where the implantation damage starts to increase rapidly (see Fig. 3). Increasing the fluence further leads to a small increase in PL intensity although the introduced damage is increasing further. Only for the highest fluence does the PL intensity decrease again. Further investigation is necessary to understand what is the nature of the detrimental defects that lead to the fast drop of PL intensity in the fluence region $(1-2) \times 10^{15}$ atoms $/ \mathrm{cm}^{2}$. In GaN a correlation of Eu emission intensity with the number of substitutional $\mathrm{Eu}$ on $\mathrm{Ga}$ sites $\left(\mathrm{Eu}_{\mathrm{Ga}}\right)$ was observed. ${ }^{24}$ In AlN, the substitutional fraction derived from RBS/C measurements decreases with fluence from about $90 \%$ for low fluences to $20 \%-30 \%$ for the highest fluences; these numbers decrease slightly after annealing possibly due to clustering of Eu with defects. At the same time the absolute number of $\mathrm{Eu}_{\mathrm{Al}}$ is increasing in the entire fluence range. This is, however, not reflected in higher PL intensities showing the strong influence of implantation damage on optical activation and nonradiative recombination.

\section{CONCLUSIONS}

We have investigated the damage buildup, annealing and optical activation of Eu implanted AIN. AIN is highly resistant to implantation damage due to its strong bonds and significant dynamic annealing. However, the implantation damage is extremely stable upon thermal annealing at temperatures as high as $1300{ }^{\circ} \mathrm{C}$ and introduces nonradiative recombination centers. Therefore it is of utmost importance to keep implantation damage low already during the implantation. Channeled implantation effectively reduces implantation damage and increases the optical activation of Eu ions.

\section{ACKNOWLEDGMENTS}

We acknowledge the support by FCT, Portugal (Grant Nos. POCI/FIS/57550/2004, PTDC/FIS/66262/2006, and PTDC/CTM/100756/2008).
${ }^{1}$ P. N. Favennec, H. L'Haridon, M. Salvi, D. Moutonnet, and Y. L. Guillou, Electron. Lett. 25, 718 (1989).

${ }^{2}$ K. P. O'Donnell and B. Hourahine, Eur. Phys. J.: Appl. Phys. 36, 91 (2006).

${ }^{3}$ A. J. Steckl, J. C. Heikenfeld, D.-S. Lee, M. J. Garter, C. C. Baker, Y. Wang, and R. Jones, IEEE J. Sel. Top. Quantum Electron. 8, 749 (2002). ${ }^{4}$ J. H. Park and A. J. Steckl, Appl. Phys. Lett. 88, 011111 (2006).

${ }^{5}$ A. Wakahara, Opt. Mater. 28, 731 (2006).

${ }^{6}$ U. Vetter, J. Zenneck, and H. Hofsäss, Appl. Phys. Lett. 83, 2145 (2003).

${ }^{7}$ J. B. Gruber, U. Vetter, H. Hofsäss, B. Zandi, and M. F. Reid, Phys. Rev. B 70, 245108 (2004).

${ }^{8}$ M. Peres, A. Cruz, M. J. Soares, A. J. Neves, T. Monteiro, K. Lorenz, and E. Alves, Superlattices Microstruct. 40, 537 (2006).

${ }^{9}$ H. J. Lozykowski and W. M. Jadwisienczak, Phys. Status Solidi B 244, 2109 (2007)

${ }^{10}$ J. M. Zavada, N. Nepal, J. Y. Lin, H. X. Jiang, E. Brown, U. Hömmerich, J. Hite, G. T. Thaler, C. R. Abernathy, S. J. Pearton, and R. Gwilliam, Appl. Phys. Lett. 89, 152107 (2006).

${ }^{11}$ N. Nepal, J. M. Zavada, D. S. Lee, and A. J. Steckl, Appl. Phys. Lett. 93, 061110 (2008).

${ }^{12}$ S. Y. Han, J. Hite, G. T. Thaler, R. M. Frazier, C. R. Abernathy, S. J. Pearton, H. K. Choi, W. O. Lee, Y. D. Park, J. M. Zavada, and R. Gwilliam, Appl. Phys. Lett. 88, 042102 (2006).

${ }^{13}$ C. Ronning, M. Dalmer, M. Uhrmacher, M. Restle, U. Vetter, L. Ziegeler, and H. Hofsäss, J. Appl. Phys. 87, 2149 (2000).

${ }^{14}$ S. O. Kucheyev, J. S. Williams, J. Zou, C. Jagadish, M. Pophristic, S. Guo, I. T. Ferguson, and M. O. Manasreh, J. Appl. Phys. 92, 3554 (2002).

${ }^{15}$ E. Wendler and W. Wesch, Nucl. Instrum. Methods Phys. Res. B 242, 562 (2006).

${ }^{16}$ W. Jiang, I.-T. Bae, and W. J. Weber, J. Phys.: Condens. Matter 19, 356207 (2007).

${ }^{17}$ F. Lu, H. Hu, and A. Rizzi, Appl. Surf. Sci. 205, 262 (2003).

${ }^{18}$ A. Vantomme, S. M. Hogg, M. F. Wu, B. Pipeleers, M. Swart, S. Goodman, D. Auret, K. Iakoubovskii, G. J. Adriaenssens, K. Jacobs, and I. Moerman, Nucl. Instrum. Methods Phys. Res. B 175-177, 148 (2001).

${ }^{19}$ B. Pipeleers, S. M. Hogg, and A. Vantomme, J. Appl. Phys. 98, 123504 (2005).

${ }^{20}$ K. Wang, K. P. O’Donnell, B. Hourahine, R. W. Martin, I. M. Watson, K. Lorenz, and E. Alves, Phys. Rev. B 80, 125206 (2009).

${ }^{21}$ K. Gärtner, K. Hehl, and G. Schlotzhauer, Nucl. Instrum. Methods 216, 275 (1983); Nucl. Instrum. Methods Phys. Res. B 4, 55 (1984); 4, 63 (1984); K. Gärtner and K. Hehl, ibid. 12, 205 (1985); K. Gärtner, ibid. 132, 147 (1997); 227, 522 (2005).

${ }^{22}$ J. F. Ziegler, J. P. Biersack, and U. Littmark, The Stopping and Range of Ions in Solids (Pergamon, New York, 1985).

${ }^{23}$ F. Gloux, T. Wojtowicz, P. Ruterana, K. Lorenz, and E. Alves, J. Appl. Phys. 100, 073520 (2006).

${ }^{24}$ K. Lorenz, N. P. Barradas, E. Alves, I. S. Roqan, E. Nogales, R. W. Martin, K. P. O'Donnell, F. Gloux, and P. Ruterana, J. Phys. D: Appl. Phys. 42, 165103 (2009). 